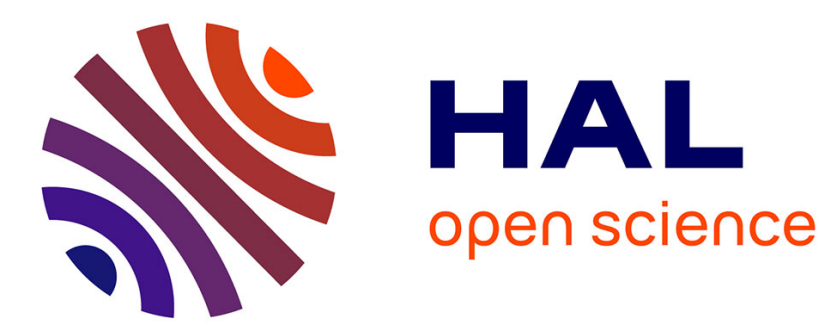

\title{
On the Chvátal-rank of facets for the set covering polyhedron of circular matrices
}

Graciela Nasini, Luis M Torres, Hervé Kerivin, Annegret K Wagler

\section{To cite this version:}

Graciela Nasini, Luis M Torres, Hervé Kerivin, Annegret K Wagler. On the Chvátal-rank of facets for the set covering polyhedron of circular matrices. Electronic Notes in Discrete Mathematics, 2018, 69, pp.85-92. 10.1016/j.endm.2018.07.012 . hal-03137958

\section{HAL Id: hal-03137958 \\ https://hal.uca.fr/hal-03137958}

Submitted on 10 Feb 2021

HAL is a multi-disciplinary open access archive for the deposit and dissemination of scientific research documents, whether they are published or not. The documents may come from teaching and research institutions in France or abroad, or from public or private research centers.
L'archive ouverte pluridisciplinaire $\mathbf{H A L}$, est destinée au dépôt et à la diffusion de documents scientifiques de niveau recherche, publiés ou non, émanant des établissements d'enseignement et de recherche français ou étrangers, des laboratoires publics ou privés. 


\title{
On the Chvátal-rank of facets for the set covering polyhedron of circular matrices*
}

\author{
Graciela Nasini \\ FCEIA, Universidad Nacional de Rosario, Rosario, Argentina \\ nasini@fceia.unr.edu.ar \\ Luis M. Torres \\ ModeMat, Escuela Politécnica Nacional, Quito, Ecuador \\ luis.torres@epn.edu.ec \\ Hervé Kerivin, Annegret Wagler \\ LIMOS, Université Clermont Auvergne, Clermont-Ferrand, France \\ \{herve.kerivin,annegret.wagler\}@uca.fr
}

February 11, 2019

\begin{abstract}
We study minor related row family inequalities for the set covering polyhedron of circular matrices. We address the issue of generating these inequalities via the Chvátal-Gomory procedure and establish a general upper bound for their Chvátal-rank. Moreover, we provide a construction to obtain facets with arbitrarily large coefficients and examples of facets having Chvátal-rank strictly larger than one.
\end{abstract}

\section{Introduction}

Given a $(m \times n)$-matrix $A$ with $(0,1)$-entries and a cost vector $c \in \mathbb{Z}^{n}$, the set packing problem (SPP) and the set covering problem (SCP) can be stated as

$$
\begin{aligned}
& (\mathrm{SPP}) \max \left\{c^{T} x: A x \leq \mathbf{1}, x \in \mathbb{Z}_{+}^{n}\right\}, \\
& (\mathrm{SCP}) \min \left\{c^{T} x: A x \geq \mathbf{1}, x \in \mathbb{Z}_{+}^{n}\right\} .
\end{aligned}
$$

Both are classic problems in combinatorial optimization with important practical applications, and are known to be hard to solve in general. One established approach to tackle such problems is to study the polyhedral properties of their

* This research was supported by the MATH-AmSud cooperation program within the framework of project PACK-COVER. 
sets of feasible solutions. The set packing polytope $P^{*}(A)$ and the set covering polyhedron $Q^{*}(A)$ are the sets defined by the convex hull of all feasible solutions of SPP and SCP, respectively. Their fractional relaxations $P(A)$ and $Q(A)$ are given by

$$
P(A):=\left\{x \in \mathbb{R}_{+}^{n}: A x \leq \mathbf{1}\right\} \text { and } Q(A):=\left\{x \in \mathbb{R}_{+}^{n}: A x \geq \mathbf{1}\right\} .
$$

In general, we have $P(A) \neq P^{*}(A)$ and $Q(A) \neq Q^{*}(A)$, even when $A$ belongs to the particular class of circular matrices, on which we focus throughout this paper. For $n \in \mathbb{N}$, let $[n]$ denote the additive group defined on the set $\{1, \ldots, n\}$, with integer addition modulo $n$. We consider the columns (resp. rows) of $A$ to be indexed by $[n]$ (resp. by $[m]$ ). $A$ is said to be circular if its rows are the incidence vectors of a set $I$ of cyclic intervals on $[n]$, with the property that no interval contains another one, i.e., $A$ has no dominating rows. A square circular matrix is called a circulant. In this case, all intervals in $I$ have the same number of elements $k$, and $I$ contains all $n$ possible intervals of this size. Thus, a circulant is completely defined by the two parameters $n$ and $k$, and we shall denote it by $C_{n}^{k}$.

The set packing polytope related to circular matrices $A$ has been studied through the equivalent stable set polytope of circular interval graphs $G$ in e.g. [7, $10,12]$. In [7] a complete linear description of $P^{*}(A)$ is obtained in terms of three families of inequalities: (i) nonnegativity constraints, (ii) clique inequalities, and (iii) the class of clique family inequalities introduced in [9]. All relevant clique family inequalities can be associated with certain induced circulant subgraphs of $G$ [12].

We are interested in studying these aspects for $Q^{*}(A)$. Valid and facetdefining inequalities for $Q^{*}(A)$ have been studied for a long time. The boolean facets include the inequalities $x \geq 0$ and $A x \geq \mathbf{1}$ defining $Q(A)$ which can be considered as the inequalities corresponding to (i) and (ii) for the set covering polyhedron. More recently, the class of row family inequalities was proposed in [3] as a counterpart of clique family inequalities in the set covering case. In fact, the same ideas used for $P^{*}(A)$ can be extended to show that $Q^{*}(A)$ is completely described by boolean facets and a particular subclass of row family inequalities associated with certain minors [5].

Given $N \subset[n]$, the minor of $A$ obtained by contraction of $N$, denoted by $A / N$, is the submatrix of $A$ that results after removing all columns with indices in $N$ and all dominating rows (whose support contains the support of one of the remaining rows). A minor of a circular matrix $A$ is called a circulant minor if it is equal to a circulant matrix $C_{n^{\prime}}^{k^{\prime}}$, up to permutation of rows and columns. We shall denote this by $A / N \approx C_{n^{\prime}}^{k^{\prime}}$.

Conditions for the existence of circulant minors of a circulant matrix have been studied in $[1,6]$. Let $G_{n}^{k}$ be a directed graph having $[n]$ as the set of nodes and all arcs of the form $(j, j+k),(j, j+k+1)$, for $1 \leq j \leq n$. Circulant minors can be characterized in terms of directed circuits in $G_{n}^{k}$.

Theorem 1 ([1]) Assume $2 \leq k \leq n-1,2 \leq n^{\prime}<n, 0<k-k^{\prime}<$ $\min \left\{k, n-n^{\prime}\right\} . C_{n}^{k} / N \approx C_{n^{\prime}}^{k^{\prime}}$ if and only if there exist $d=\operatorname{gcd}\left(n-n^{\prime}, k-k^{\prime}\right)$ 
disjoint simple directed circuits in $G_{n}^{k}, D_{1}, \ldots, D_{d}$, each having length $\frac{n-n^{\prime}}{d}$, such that $N=\cup_{t=1}^{d} V\left(D_{t}\right)$.

Circulant minors of $C_{n}^{k}$ are known to induce valid (and in some cases facetdefining) inequalities for $Q^{*}\left(C_{n}^{k}\right)$. The class of minor inequalities was introduced in [2] and was further studied and generalized in [4, 13]. In [5] it was observed that a circulant minor $C_{n}^{k} / N \approx C_{n^{\prime}}^{k^{\prime}}$ also induces a row family inequality that either is equivalent to or enhances the corresponding minor inequality. This minor related row family inequality (minor $\mathrm{rfi}$ ) has the form

$$
r \sum_{j \notin W} x_{j}+(r+1) \sum_{j \in W} x_{j} \geq r\left\lceil\frac{n^{\prime}}{k^{\prime}}\right\rceil,
$$

where $r=n^{\prime} \bmod k^{\prime}$, and $W \subset N$ is the set of nodes in the circuits $D_{t}$ that are heads of arcs of the form $(j, j+k+1)$.

In [4] it was conjectured that $(0,1)$ - and $(1,2)$-valued minor inequalities suffice to describe $Q^{*}\left(C_{n}^{k}\right)$. This was disproved in [13], where a first example of a facet-defining $(2,3)$-valued minor inequality is presented. Using similar ideas as for the case of $P^{*}\left(C_{n}^{k}\right)$ in [8], in this paper we show that there are circulant matrices such that $Q^{*}\left(C_{n}^{k}\right)$ has facet-defining minor related row family inequalities with two consecutive arbitrarily large coefficients.

Moreover, we are interested in studying the difference between $Q(A)$ and $Q^{*}(A)$ in terms of the Chvátal-Gomory procedure. For given $a \in \mathbb{Z}^{n}$ and $b \notin \mathbb{Z}$, assume $a^{T} x \geq b$ is valid for $Q(A)$ and tight for some $x^{*} \in Q(A)$. Then the inequality $a^{T} x \geq\lceil b\rceil$ is valid for $Q^{*}(A)$, but violated by $x^{*}$. Such an inequality is called a Chvátal-Gomory cut for $Q(A)$ and the procedure for obtaining it is the Chvátal-Gomory procedure. The first Chvátal closure $Q^{\prime}(A)$ is the set of points of $Q(A)$ satisfying all Chvátal-Gomory cuts. Let $Q^{0}:=Q(A)$ and $Q^{t}:=\left(Q^{t-1}\right)^{\prime}$ for all $t \in \mathbb{N}$. Evidently, $Q^{*}(A) \subseteq Q^{t} \subseteq Q^{t-1}$ holds for every $t \in \mathbb{N}$. Moreover, it is known that there exists a finite $\hat{t} \in \mathbb{N}$ with $Q^{\hat{t}}=Q^{*}(A)$; the smallest such $\hat{t}$ is the Chvátal-rank of $Q(A)$. An inequality is said to have Chvátal-depth equal to $t$ if it is valid for $Q^{t}$, but not valid for $Q^{t-1}$.

The Chvátal-rank of $Q(A)$ has been addressed in several previous works. Any minor inequality has Chvátal-depth at most one, but it has been observed in [3] that this might not be the case for row family inequalities.

In the context of set packing, a general upper bound has been presented in [11] for the Chvátal-depth of clique family inequalities. In this paper, we follow a similar approach to establish a general upper bound for the Chvátal-depth

of minor rfi's, and provide examples of minor rfi's with Chvátal-depth strictly larger than one.

\section{Facets with arbitrarily large coefficients}

In the following we provide a construction to show that minor related row family inequalities with arbitrarily large coefficients can occur as facets of $Q^{*}(A)$, even in the particular case when $A$ is a circulant matrix $C_{n}^{k}$. 
Let $\alpha \in \mathbb{N}$ with $\alpha \geq 6$ and define $n:=(\alpha-1)(\alpha+1), k:=\alpha$. Moreover, consider the finite sequences of natural numbers given by

$$
n_{r}:=(\alpha-1)(\alpha-r) \quad k_{r}:=\alpha-r-1,
$$

where $r$ takes values from the set $S:=\left\{1, \ldots,\left\lfloor\frac{\alpha}{2}\right\rfloor-1\right\}$. It is straightforward to verify that $C_{n_{r}}^{k_{r}}$ is a circulant minor of $C_{n}^{k}$, for all $r \in S$. Indeed, the conditions of Theorem 1 are satisfied as $G_{n}^{k}$ contains $d=r+1$ disjoint simple directed circuits, each one consisting of $\alpha-1$ arcs of length $k+1$ and no arcs of length $k$. Let $W_{r}$ denote the union of the sets of nodes of these circuits. Moreover, since $2 r+1<\alpha$, it follows that $r<\alpha-r-1$ and

$$
\frac{n_{r}}{k_{r}}=\frac{(\alpha-1)(\alpha-r)}{\alpha-r-1}=\alpha+\frac{r}{\alpha-r-1}<\alpha+1 .
$$

Hence, $\left\lceil\frac{n_{r}}{k_{r}}\right\rceil=\alpha+1$ and $n_{r}=r \bmod k_{r}, \forall r \in S$. The minor related row family inequality of $Q^{*}\left(C_{n}^{k}\right)$ induced by $C_{n_{r}}^{k_{r}}$ is

$$
r \sum_{j \notin W_{r}} x_{j}+(r+1) \sum_{j \in W_{r}} x_{j} \geq r(\alpha+1) .
$$

Theorem 2 Inequality (2) defines a facet of $Q^{*}\left(C_{n}^{k}\right)$ if $\operatorname{gcd}(r, \alpha-1)=1$.

In particular, if $\alpha-1$ is a prime number then $Q^{*}\left(C_{\alpha^{2}-1}^{\alpha}\right)$ has facets steming from minor rfi's with all possible coefficients $r, r+1$, for $1 \leq r \leq\left\lfloor\frac{\alpha}{2}\right\rfloor-1$.

Example 3 Choosing $\alpha=8$, we obtain that $C_{63}^{8}$ contains all circulant minors of the form $C_{7(8-r)}^{7-r}$ with $r \in\{1,2,3\}$. As $\alpha-1$ is prime, these minors $C_{49}^{6}$, $C_{42}^{5}, C_{35}^{4}$ induce $(r, r+1)$-valued facets of $Q^{*}\left(C_{63}^{8}\right)$.

Moreover, with the help of Theorem 2, we can construct sequences of circulants and (sub)circulant minors such that the associated minor rfi's have arbitrarily high coefficients:

Example 4 For each $r \geq 2$ and $\alpha=2(r+1)$, the row family inequality associated with the minor $C_{(2 r+1)(r+2)}^{r+1}$ of $C_{(2 r+1)(2 r+3)}^{2 r+2}$ induces an $(r, r+1)$-valued facet of $Q^{*}\left(C_{(2 r+1)(2 r+3)}^{2 r+2}\right)$, since $\operatorname{gcd}(r, \alpha-1)=\operatorname{gcd}(r, 2 r+1)=1$.

Example 5 For each $r \geq 2$ and $\alpha=3 r+2$, the row family inequality associated with the minor $C_{(3 r+1)(2 r+2)}^{2 r+1}$ of $C_{(3 r+1)(3 r+3)}^{3 r+2}$ induces an $(r, r+1)$-valued facet of $Q^{*}\left(C_{(3 r+1)(3 r+3)}^{3 r+2}\right)$, since $\operatorname{gcd}(r, \alpha-1)=\operatorname{gcd}(r, 3 r+1)=1$.

Corollary 6 There exist infinite families of circulants $C_{n}^{k}$ for which $Q^{*}\left(C_{n}^{k}\right)$ has facets with two arbitrarily large consecutive coefficients. 


\section{On the Chvátal-depth of minor rfi's}

Here, we study the Chvátal-depth of the above defined minor rfi's and address bounds for their Chvátal-depth.

Upper bounds. In a similar spirit as upper bounds for the Chvátal-depth of clique family inequalities where obtained in [11], we establish here one general upper bound for the Chvátal-depth of any minor rfi

$$
r \sum_{j \notin W} x_{j}+(r+1) \sum_{j \in W} x_{j} \geq r\left\lceil\frac{n^{\prime}}{k^{\prime}}\right\rceil
$$

with $r=n^{\prime} \bmod k^{\prime}$ induced by a circulant minor $C_{n^{\prime}}^{k^{\prime}}$ of some circulant matrix $C_{n}^{k}$. Consider the inequality obtained by adding all rows corresponding to the circulant minor, together with (possibly) some non-negativity constraints. This inequality is valid for $Q(A)$ and has the form

$$
k^{\prime} \sum_{j \notin W} x_{j}+\left(k^{\prime}+1\right) \sum_{j \in W} x_{j} \geq n^{\prime}=k^{\prime}\left\lceil\frac{n^{\prime}}{k^{\prime}}\right\rceil-k^{\prime}+r .
$$

Furthermore, for $i=0, \ldots, r$, let $h(i)$ be the inequality

$$
h(i): \quad i \sum_{j \notin W} x_{j}+(i+1) \sum_{j \in W} x_{j} \geq i\left\lceil\frac{n^{\prime}}{k^{\prime}}\right\rceil .
$$

Observe that $h(0)$ is valid for $Q(A)$, as it is a combination of non-negativity constraints, while $h(r)$ is precisely the minor rfi (1). We can prove:

Lemma 7 For $i \in\{1, \ldots, r\}$, inequality $h(i)$ can be obtained from (3) and $h(i-1)$ with a single application of the Chvátal-Gomory procedure.

Moreover, for $k^{\prime}-r \leq r$, denote by $g(i)$ the following inequality

$$
g(i): \quad\left(k^{\prime}-i\right) \sum_{j \notin W} x_{j}+\left(k^{\prime}-i+1\right) \sum_{j \in W} x_{j} \geq\left(k^{\prime}-i\right)\left\lceil\frac{n^{\prime}}{k^{\prime}}\right\rceil-k^{\prime}+r+i .
$$

Observe that $g(0)$ coincides with inequality (3) and is therefore valid for $Q(A)$, while $g\left(k^{\prime}-r\right)$ is precisely the minor rfi (1). We can prove the following:

Lemma 8 For $i \in\left\{1, \ldots, k^{\prime}-r\right\}$, inequality $g(i)$ can be obtained from $h(i-1)$ and $g(i-1)$ with a single application of the Chvátal-Gomory procedure.

As a consequence from Lemma 7 and Lemma 8, we obtain the following.

Theorem 9 The Chvátal-depth of the minor rfi (1) induced by a circulant minor $C_{n^{\prime}}^{k^{\prime}}$ of some circulant matrix $C_{n}^{k}$ is bounded from above by $\min \left\{r, k^{\prime}-r\right\}$ where $r=n^{\prime} \bmod k^{\prime}$. 
This shows, for instance, that the minor rfi's with arbitrarily high coefficients $r, r+1$ for any $r \geq 2$ from Example 4 have only Chvátal-depth 1 by $\min \left\{r, k^{\prime}-r\right\}=\min \{r,(r+1)-r\}=1$, whereas the minor rfi's from Example 5 have Chvátal-depth at most $r$ by $\min \left\{r, k^{\prime}-r\right\}=\min \{r,(2 r+1)-r\}=r$ (and, thus may have arbitrarily high Chvátal-depth).

Lower bounds. It is open to establish lower bounds on the Chvátal-depth of minor rfi's for general circular matrices $A$. The following lemma provides a condition for a minor rfi to have Chvátal-depth strictly larger than one when $A$ is a circulant.

Lemma 10 If $r^{2}<(\alpha-r-1)(r-1)$ then the inequality (2) induced by $C_{n_{r}}^{k_{r}}$ cannot be obtained from the inequalities in the system defining $Q\left(C_{n}^{k}\right)$ by a single application of the Chvátal-Gomory procedure.

The last result does not necessarily imply that the inequality induced by the minor $C_{n_{r}}^{k_{r}}$ has Chvátal-depth larger than one, as it can still be obtained as a nonnegative combination of other inequalities with Chvátal-depth equal to one. However, this cannot be the case if the studied inequality defines a facet of $Q^{*}\left(C_{n}^{k}\right)$. Together with Theorem 2, this implies:

Theorem 11 If $(\alpha-r-1)(r-1)>r^{2}$ and $\operatorname{gcd}(r, \alpha-1)=1$ then the inequality (2) induced by $C_{n_{r}}^{k_{r}}$ has Chvátal-depth strictly larger than one.

In particular, choosing $r=2$ it follows that $Q\left(C_{\alpha^{2}-1}^{\alpha}\right)$ has Chvátal-rank strictly larger than one for all even $\alpha \geq 8$. The smallest such example with $r=2$ and $\alpha=8$ is $Q\left(C_{63}^{8}\right)$, as $Q^{*}\left(C_{63}^{8}\right)$ has a facet with Chvátal-depth larger than one induced by the minor $C_{42}^{5}$.

This lower bound also applies to the $(r, r+1)$-valued minor rfi's from Example 5 , since $r^{2}<k_{r}(r-1)=(2 r+1)(r-1)=2 r^{2}-r-1$ holds by $r+1<r^{2} \forall r \geq 2$ and shows that these minor rfi's have Chvátal-depth strictly larger than one (and are potential examples of minor rfi's with arbitrarily high Chvátal-depth in view of the upper bound of $r$ ).

\section{Concluding remarks}

In this paper, we studied the family of minor related row family inequalities for the set covering polyhedron related to circular matrices. We addressed the issue of generating these inequalities via the Chvátal-Gomory procedure and established a general upper bound for their Chvátal-depth. Moreover, we provided a construction for facets of $Q^{*}\left(C_{n}^{k}\right)$ with arbitrarily large coefficients, belonging to the class of minor rfi's. We presented examples of $(r, r+1)$-valued facets of this type with Chvátal-depth bounded from above by 1 and $r$, where the latter have Chvátal-depth strictly larger than one. This shows that, in this respect, minor rfi's differ from other previously described minor induced inequalities for $Q^{*}\left(C_{n}^{k}\right)$, which are known to have Chvátal-depth at most one. As future work, 
we intend to investigate whether larger lower bounds on the Chvátal-depth can be proven for inequalities with large coefficients.

Acknowledgement. Silvia Bianchi from Universidad Nacional de Rosario has independently found another construction to obtain facets of $Q^{*}\left(C_{n}^{k}\right)$ with arbitrarily large coefficients. We thank her for many fruitful discussions.

\section{References}

[1] Aguilera, N., On packing and covering polyhedra of consecutive ones circulant clutters, Discrete Applied Mathematics 158 (2009), pp. 1343-1356.

[2] Argiroffo, G. and S. Bianchi, On the set covering polyhedron of circulant matrices, Discrete Optimization 6 (2009), pp. 162-173.

[3] Argiroffo, G. and S. Bianchi, Row family inequalities for the set covering polyhedron, Electronic Notes in Discrete Mathematics 36 (2010), pp. 1169-1176.

[4] Bianchi, S., G. Nasini and P. Tolomei, The set covering problem on circulant matrices: polynomial instances and the relation with the dominating set problem on webs, Electronic Notes in Discrete Mathematics 36 (2010), pp. 1185-1192.

[5] Bianchi, S., G. Nasini, P. Tolomei and L. M. Torres, On dominating set polyhedra of circular interval graphs, Preprint arXiv:1712.07057 (2017).

[6] Cornuéjols, G. and B. Novick, Ideal $0-1$ matrices, Journal of Combinatorial Theory (B) 60 (1994), pp. 145-157.

[7] Eisenbrand, F., G. Oriolo, G. Stauffer and P. Ventura, The stable set polytope of quasi-line graphs, Combinatorica 28 (2008), pp. 45-67.

[8] Liebling, T. M., G. Oriolo, B. Spille and G. Stauffer, On non-rank facets of the stable set polytope of claw-free graphs and circulant graphs, Math. Meth. Oper. Res. 59 (2004), pp. 25-35.

[9] Oriolo, G., Clique family inequalities for the stable set polytope for quasi-line graphs, Discrete Applied Mathematics 132 (2003), pp. 185-201.

[10] Pêcher, A. and A. Wagler, Almost all webs are not rank-perfect, Math. Prog. 105 (2006), pp. 311-328.

[11] Pêcher, A. and A. Wagler, A note on the chvátal-rank of clique family inequalities, RAIRO Oper. Res. 41 (2007), pp. 289-294.

[12] Stauffer, G., "On the Stable Set Polytope of Claw-free Graphs," Ph.D. thesis, EPF Lausanne (2005).

[13] Tolomei, P. and L. M. Torres, Generalized minor inequalities for the set covering polyhedron related to circulant matrices, Discrete Applied Mathematics 210 (2016), pp. 214-222. 